\title{
Chaos-Based Optimization - A Review
}

\author{
Roman SENKERIK ${ }^{1,2, *}$, Ivan ZELINKA ${ }^{1,3}$, Michal PLUHACEK ${ }^{2}$
}

\author{
${ }^{1}$ Modeling Evolutionary Algorithms Simulation and Artificial Intelligence, Faculty of Electrical \\ and Electronics Engineering, Ton Duc Thang University, Ho Chi Minh City, Vietnam \\ ${ }^{2}$ Faculty of Applied Informatics, Tomas Bata University in Zlin, Nam T.G. Masaryka 5555, 760 \\ 01 Zlin, Czech Republic \\ ${ }^{3}$ Technical University of Ostrava, Faculty of Electrical Engineering and Computer Science, 17. \\ listopadu 15, 70833 Ostrava-Poruba, Czech Republic \\ *roman.senkerik@tdt.edu.vn; senkerik@fai.utb.cz
}

(Received: 11-February-2017; accepted: 3-May-2017; published: 8-June-2017)

DOI: http://dx.doi.org/10.25073/jaec.201711.51

\begin{abstract}
This paper discusses the utilization of the complex chaotic dynamics given by the selected time-continuous chaotic systems as well as by the discrete chaotic maps, as the chaotic pseudo-random number generators and driving maps for the chaos based optimization. Such an optimization concept is utilizing direct output iterations of chaotic system transferred into the required numerical range as the replacement of traditional and default pseudo-random number generators, or this concept uses the chaotic dynamics for mapping the search space mostly within the smart hybrid local search techniques. This paper shows totally three groups of complex chaotic dynamics given by chaotic flows, oscillators and discrete maps. Simulations of examples of chaotic dynamics as unconventional generators or mapped to the search space were performed, and related issues like parametric plots, distributions of such a systems, periodicity, and dependency on internally available parameters are briefly discussed in this paper.
\end{abstract}

\section{Keywords}

Deterministic chaos, Heuristic, Chaotic Optimization, Chaotic Pseudo Random Number Generators.

\section{Introduction}

Generally speaking, the term "chaos" can denote anything that cannot be predicted deterministically. In the case that the word "chaos" is combined with an attribute such as "deterministic," then a specific type of chaotic phenomena is involved, having their specific laws, mathematical apparatus and a physical origin. The deterministic chaos is a phenomenon that - as its name suggests - is not based on the presence of a random or any stochastic effects. It is evident from the structure of the equations (see the section: Chaotic Optimization) that no mathematical term expressing randomness is present. The seeming randomness in deterministic chaos is related to the extreme sensitivity to the initial conditions [1].

In the past, the chaos has been observed in many of various systems (including evolutionary one). Systems exhibiting deterministic chaos include, for instance, weather, biological systems, many electronic circuits (Chua's circuit), mechanical systems, such as a double pendulum, magnetic pendulum, or so-called billiard problem. The idea of using chaotic systems instead of random processes (pseudo-number generators - PRNGs) has been presented in several research fields and many applications with promising results [2], [3]. 
Another research joining deterministic chaos and pseudorandom number generator has been done for example in [4]. The possibility of generation of random or pseudorandom numbers by use of the ultra-weak multidimensional coupling of $\mathrm{p}$ 1-dimensional dynamical systems is discussed there.

Another paper [5], deeply investigate logistic map as a possible pseudorandom number generator and is compared with contemporary pseudo-random number generators. A comparison of logistic map results is made with conventional methods of generating pseudorandom numbers. The approach used to determine the number, delay, and period of the orbits of the logistic map at varying degrees of precision (3 to 23 bits). Another paper [6] proposed an algorithm for generating pseudorandom number generator, which is called (coupled map lattice based on discrete chaotic iteration) and combine the coupled map lattice and chaotic iteration. Authors also tested this algorithm in NIST 80022 statistical test suits and for future utilization in image encryption. In (Narendra et al. 2010) authors exploit interesting properties of chaotic systems to design a random bit generator, called CCCBG, in which two chaotic systems are crosscoupled with each other. A new binary stream cipher algorithm based on dual one-dimensional chaotic maps is proposed in [7] with statistic proprieties showing that the sequence is of high randomness. Similar studies are also done in [8].

\section{Motivation}

This paper represents the extension of preliminary suggestions described in [9. Recently the deterministic chaos has frequently been used either as a replacement of (mostly uniform distribution based) pseudo-number generators (PRGNs) in metaheuristic algorithms or for simple mapping of solutions/iterations within original local search engines. The metaheuristic chaotic approach uses the chaotic system in the place of a pseudo-random number generator [10]. This method causes the heuristic to map search regions based on unique sequencing and periodicity of transferred chaotic dynamics, thus simulating the dynamical alternations of several sub- populations. The task is then to select an appropriate chaotic system (either discrete or timecontinuous) as the chaotic pseudo random number generator (CPRNG) [11.

Recently, the concept of embedding of chaotic dynamics into the evolutionary algorithms has been studied intensively. The self-adaptive chaos differential evolution (SACDE) [12] was followed by the implementation of chaos into the simple not-adaptive differential evolution [13], 14]; the chaotic searching algorithm for the very same metaheuristic was introduced in [15]. Also, the PSO (Particle Swarm Optimization) algorithm with elements of chaos was introduced as CPSO [16]. Many other works focusing on the hybridization of the swarm and chaotic movement have been published afterward [17, [18]. Later on, the utilization of chaotic sequences became to be popular in many interdisciplinary applications and techniques. The question of impact and importance of different randomization within heuristic search was intensively studied in [19].

The primary aim of this work is to try, test, analyze and compare the implementation of different natural chaotic dynamic either as the mapping procedure for the optimization/searching process or as the CPRNG. This paper presents the discussion about the usability of such systems, periodicity, and dependency on accessible internal parameters; thus the usability as CPRNG, or for local search and metaheuristic based optimization techniques.

\section{Chaotic Optimization}

There exist three possible utilizations of chaotic dynamics in optimization tasks.

Firstly, as aforementioned in the previous section, the direct output simulation iterations of the chaotic system are transferred into the required numerical range (as simple CPRNG). The idea of CPRNG is to replace the default system PRNG with the chaotic system. As the chaotic system is a set of equations with a static start position (see the next section), we created a random start position of the system, to have different start position for various experiments. 
Once the origin position of the chaotic system has been obtained, the system generates the next sequence using its current position. Subsequently, simple techniques as to how to deal with the negative numbers as well as with the scaling of the wide range of the numbers given by the chaotic systems into the typical range $0-1$.

Secondly, the complexity of chaotic systems and its movement in the space is used for dynamical mapping of the search space mostly within the local search techniques [20. Finally, the hybridization of searching/optimization process and chaotic systems is represented by chaos based random walk technique.

\section{Chaotic Optimization}

This section contains the description of three different groups of chaotic dynamics: timecontinuous chaotic systems (flows and oscillators), and the discrete chaotic maps.

\subsection{Time Continuous systems as CPRNG}

In this research, following chaotic systems were used: Lorenz system (1) and Rossler system (2) as two examples of chaotic flows; further unmodified UEDA oscillator (3); and Driven Van der Pol Oscillator (4) as chaotic oscillators [21.

The Lorenz system (1) is a 3-dimensional dynamical flow, which exhibits chaotic behavior. It was introduced in 1963 by Edward Lorenz, who derived it from the simplified equations of convection rolls arising in the equations of the atmosphere.

The Rossler system (2) exhibits chaotic dynamics associated with the fractal properties of the attractor. It was originally introduced as an example of very simple chaotic flow containing chaos similarly to the Lorenz attractor. This attractor has some similarities to the Lorenz attractor but is simpler.

UEDA oscillator (3) is the simple example of driven pendulums, which represent some of the most significant examples of chaos and regularity.

UEDA system can be simply considered as a special case of intensively studied Duffing oscillator that has both a linear and cubic restoring force. Ueda oscillator represents the both biologically and physically relevant dynamical model exhibiting chaotic motion.

Finally, The Van der Pol oscillator (4) is the simple example of the limit cycles and chaotic behavior in electrical circuits employing vacuum tubes. Similarly to the UEDA oscillator, it can be used to explore physical (unstable) behavior in biological sciences. [22].

The equations, which describe the chaotic systems, have parameter settings for Lorenz system: $a=3.0, b=26.5$; Rossler system: $a=0.2$, $b=0.2$, and $c=5.7$; UEDA oscillator: $a=1.0$, $b=0.05, c=7.5$ and $\omega=1.0$; and Van der Pol oscillator : $\mu=0.2, \gamma=8.0, a=0.35$ and $\omega=1.02$.

$$
\begin{aligned}
& \frac{d x}{d t}=-a(x-y) \\
& \frac{d y}{d t}=x(b-z)-y . \\
& \frac{d z}{d t}=x y-z
\end{aligned}
$$

$$
\begin{aligned}
& \frac{d x}{d t}=-x-y \\
& \frac{d y}{d t}=x+a y . \\
& \frac{d z}{d t}=b+z(x-c)
\end{aligned}
$$

$$
\begin{aligned}
& \frac{d x}{d t}=y \\
& \frac{d y}{d t}=-a x^{3}-b y+c \sin \omega t
\end{aligned}
$$

$$
\begin{aligned}
& \frac{d x}{d t}=y \\
& \frac{d y}{d t}=\mu\left(1-\gamma x^{2}\right) y-x^{3}+a \sin \omega t
\end{aligned}
$$


The parametric plots of the chaotic systems are depicted in Fig. 1. The Fig. 2 show the example of dynamical sequencing during the generating of pseudo random numbers transferred into the range $<0-1>$ using particular studied CPRNGs and with the sampling rate of $0.5 \mathrm{~s}$. The dependency of sequencing and periodicity on the sampling frequency is discussed in details in 23. Finally, the comparison of CPRNGs distribution is depicted in Fig. 3 .

\subsection{Discrete Chaotic Maps as CPRNG}

The examples of chaotic maps are following: Arnold Cat map (5), Dissipative Standard map (6), Ikeda map (7), and Lozi map (8). Map equations and parameters values are given in Table 1, as suggested in [21]. Parametric plots are depicted in Fig. 44 whereas the examples of dynamical sequencing are given in Fig. 4 and distribution histograms for 5000 samples of CPRNG sequences are in Fig. 6 .

\subsection{Discrete Chaotic Maps for Mapping of Search Space}

Finally, this last subsection discusses the utilization of discrete chaotic maps for effective dynamical mapping of the search space suitable for either random/local search or random walk based engines. The reason, as to why the discrete maps were chosen for the mapping experiment is given in the previous subsection, where the selected discrete chaotic maps are depicted in graphics grid (Fig. 3) and sorted by the highest density of coverage to the least. The graphical data in Fig. 4 lends weight to the argument that some chaotic maps support the basic claim and feature of deterministic chaos. This feature is called density of periodic orbits, assuming that chaotic attractor (system) will visit most of the points in the space.

Furthermore presented simple discrete chaotic systems have additional accessible parameters (See Tab.1.), which can by tuned. This issue opens up the possibility of examining the impact of these parameters on the generation of ran- dom numbers and thus influence on chaos-based optimization (including adaptive switching between chaotic systems or sampling rates). The impact of parameter changing for the chaotic map is demonstrated in Fig. 7, Fig. 8, Fig. 9 Fig. 10, Fig. 11, Fig. 12, Fig. 13, Slight change of original settings for Dissipative map resulted in increasing of the search space mapping density (See Fig. 9 compared to the Fig. 4). The change of chaotic dynamics is also transferred to different search trajectories (Figs 7 and 8 compared to the Fig. 10 and Fig. 11). Such a change in density of search space coverage and change in dynamical steps is also demonstrated in two histograms of density: Fig. 12 and Fig. 13.

\subsection{Simulation Results Discussion}

The main aim of this research is to try, test, analyze the usability and compare the implementation of different natural chaotic dynamic either as the replacement of traditional/default PRNGs or as the mapping procedure for the optimization/searching process. Eight different complex chaotic time-continuous flows/oscillators and discrete chaotic maps have been simulated, and the output behaviors have been transferred into the pseudo-random number sequences. Findings can be summarized in Tab. 2 and expanded as follows:

- In many research works, it was proven that chaos based optimization is very sensitive to the hidden chaotic dynamics driving the CPRNG/mapping the search space. Such a chaotic dynamics can be significantly changed by the selection of sampling time in the case of the time-continuous systems (both flows and oscillators). A small sampling rate of approx. $0.1 \mathrm{~s}-0.5 \mathrm{~s}$ keeps the information about the chaotic dynamics inside the generated pseudo-random sequence. By changing or simple learning adaptation of such sampling frequency, we can fully keep, partially suppress or even fully remove the chaotic information from CPRNG sequence. There is no such a control possibility for discrete maps. 

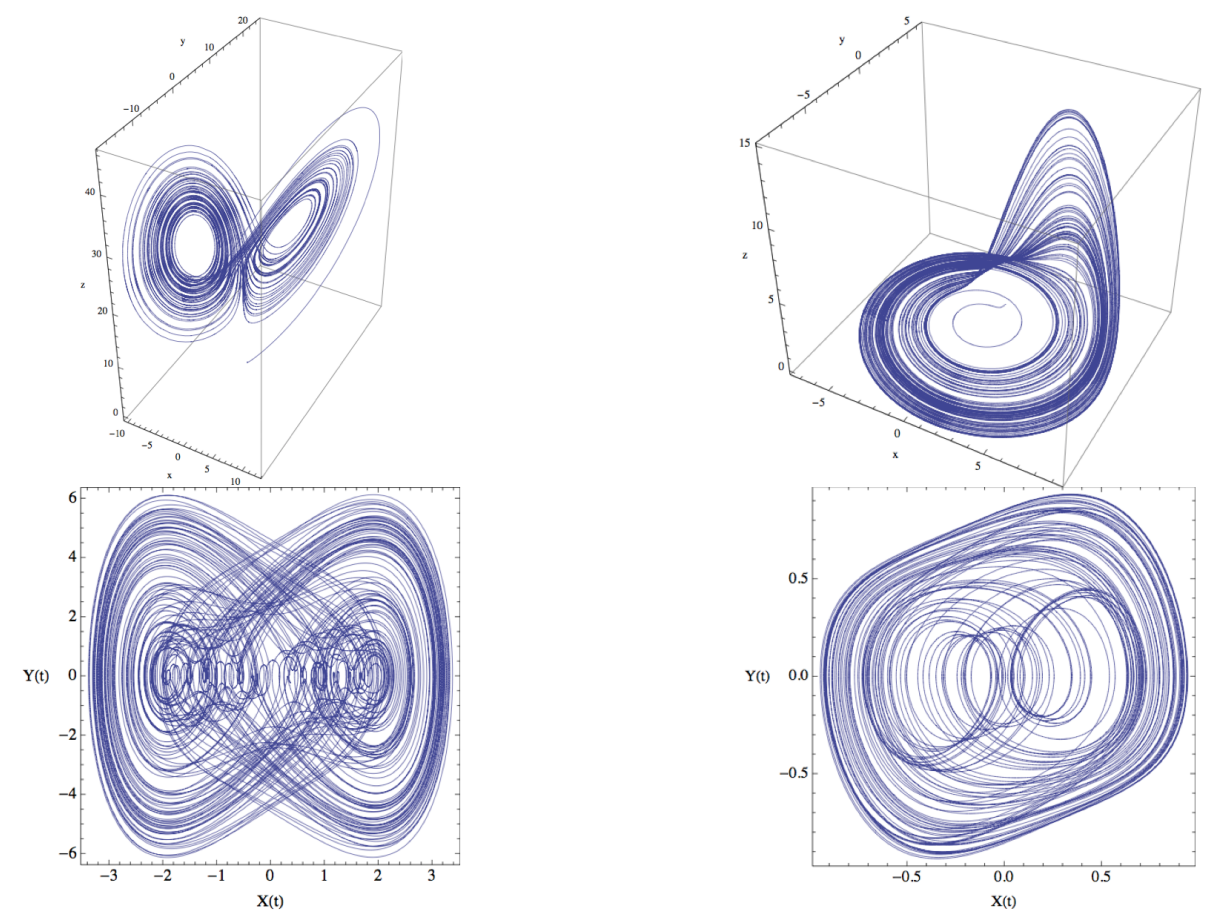

Fig. 1: Parametric plots of time-continuous chaotic systems; upper left - Lorenz system, upper right - Rossler system, bottom left - UEDA oscillator, bottom right - Van der Pol Oscillator.
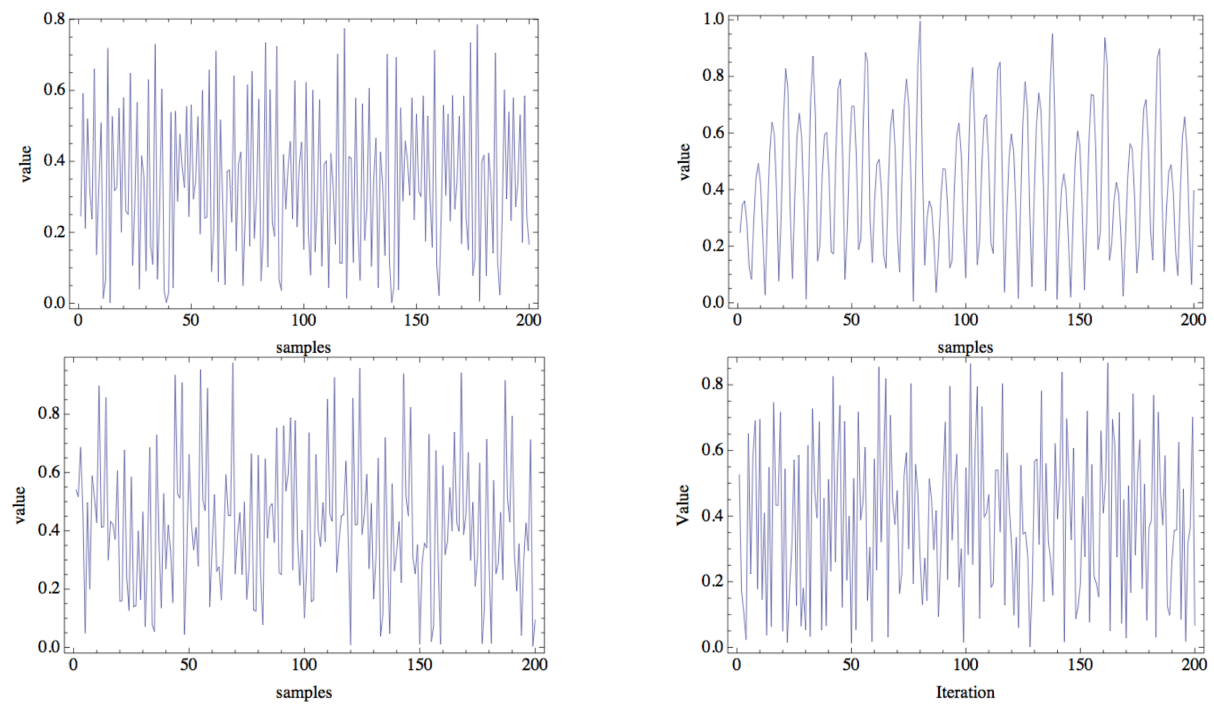

Fig. 2: Comparison of the dynamical sequencing of pseudo-random real numbers transferred into the range $<$ $0-1>$; upper left ? Lorenz system, upper right Rossler system, bottom left UEDA oscillator, bottom right Van der Pol Oscillator. 

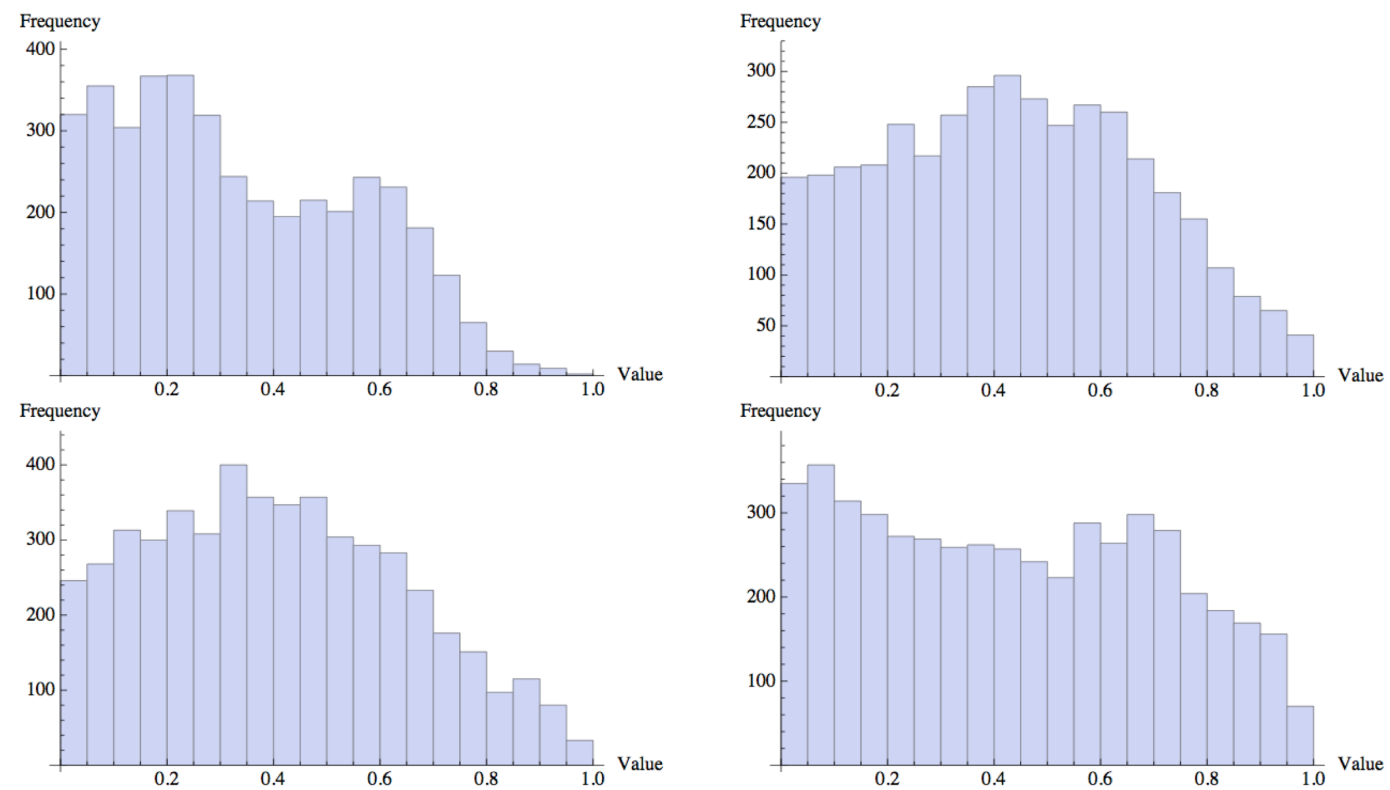

Fig. 3: Comparison of distributions of pseudo-random real numbers transferred into the range $<0-1>(5000$ samples); upper left ? Lorenz system, upper right Rossler system, bottom left UEDA oscillator, bottom right Van der Pol Oscillator.

Tab. 1: Used discrete chaotic systems as CPRNG and parameters set up.

\begin{tabular}{|c|c|c|}
\hline Chaotic system & Notation & Parameters values \\
\hline Arnold Cat Map & $\begin{array}{l}X_{n+1}=X_{n}+Y_{n}(\bmod 1) \\
Y_{n+1}=X_{n}+k Y_{n}(\bmod 1)\end{array}$ & $k=2.0$ \\
\hline Dissipative Standard Map & $\begin{array}{l}X_{n+1}=X_{n}+Y_{n+1}(\bmod 2 \pi) \\
Y_{n+1}=b Y_{n}+k \sin X_{n}(\bmod 2 \pi)\end{array}$ & $b=0.6$ and $k=8.8$ \\
\hline Ikeda Map & $\begin{array}{l}X_{n+1}=\gamma+\mu\left(X_{n} \cos \phi+Y_{n} \sin \phi\right) \\
Y_{n+1}=\mu\left(X_{n} \sin \phi+Y_{n} \cos \phi\right) \\
\phi=\beta-\alpha /\left(1+X_{n}^{2}+Y_{n}^{2}\right)\end{array}$ & $\alpha=6, \beta=0.4, \gamma=1$ and $\mu=0.9$ \\
\hline Lozi Map & $\begin{array}{l}X_{n+1}=1-a\left|X_{n}\right|+b Y_{n} \\
Y_{n+1}=X_{n}\end{array}$ & $a=1.7$ and $b=0.5$ \\
\hline
\end{tabular}



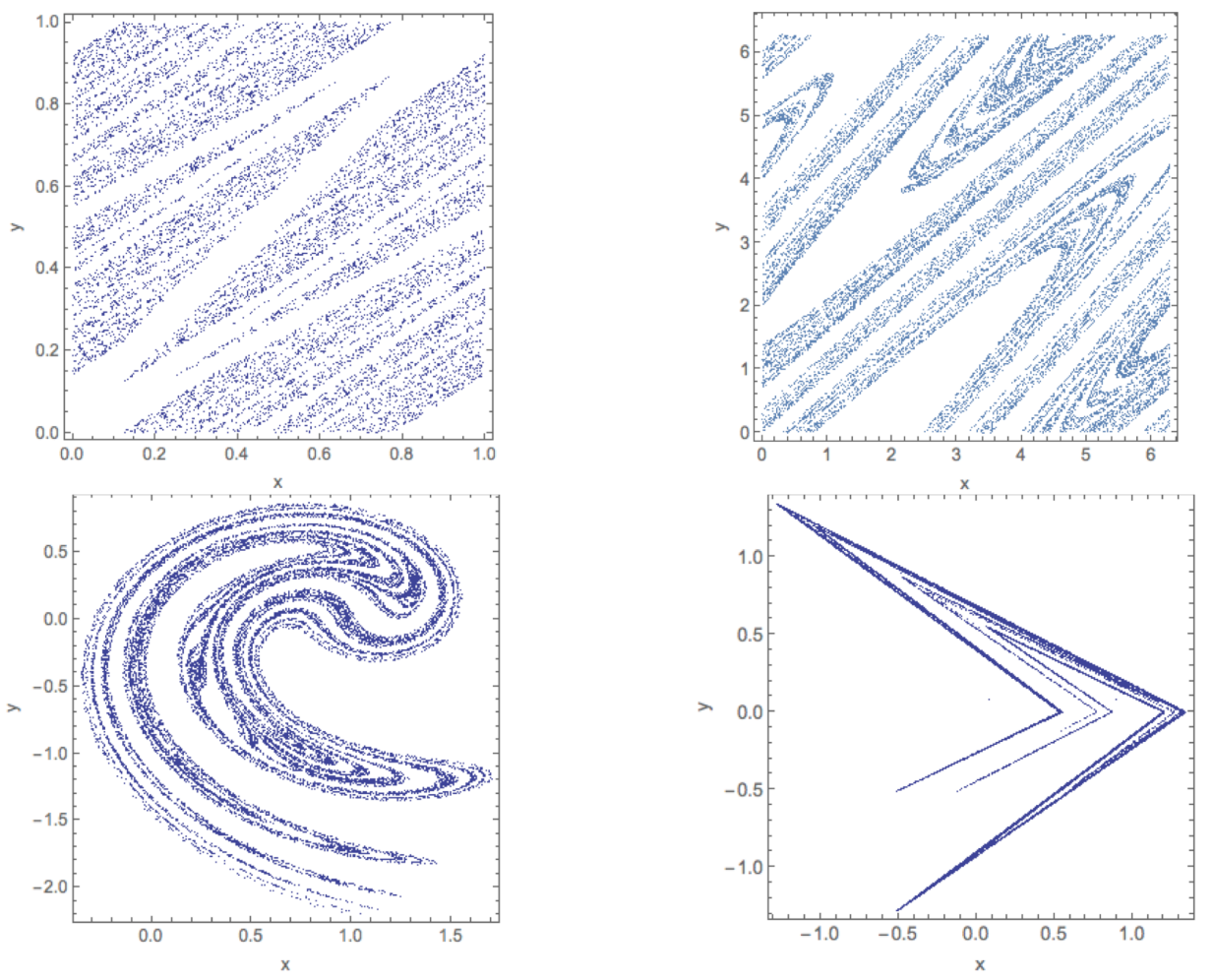

Fig. 4: Parametric plots of discrete chaotic maps; upper left - Arnold Cat map, upper right Dissipative standard map, bottom left Ikeda map, bottom right Lozi map.
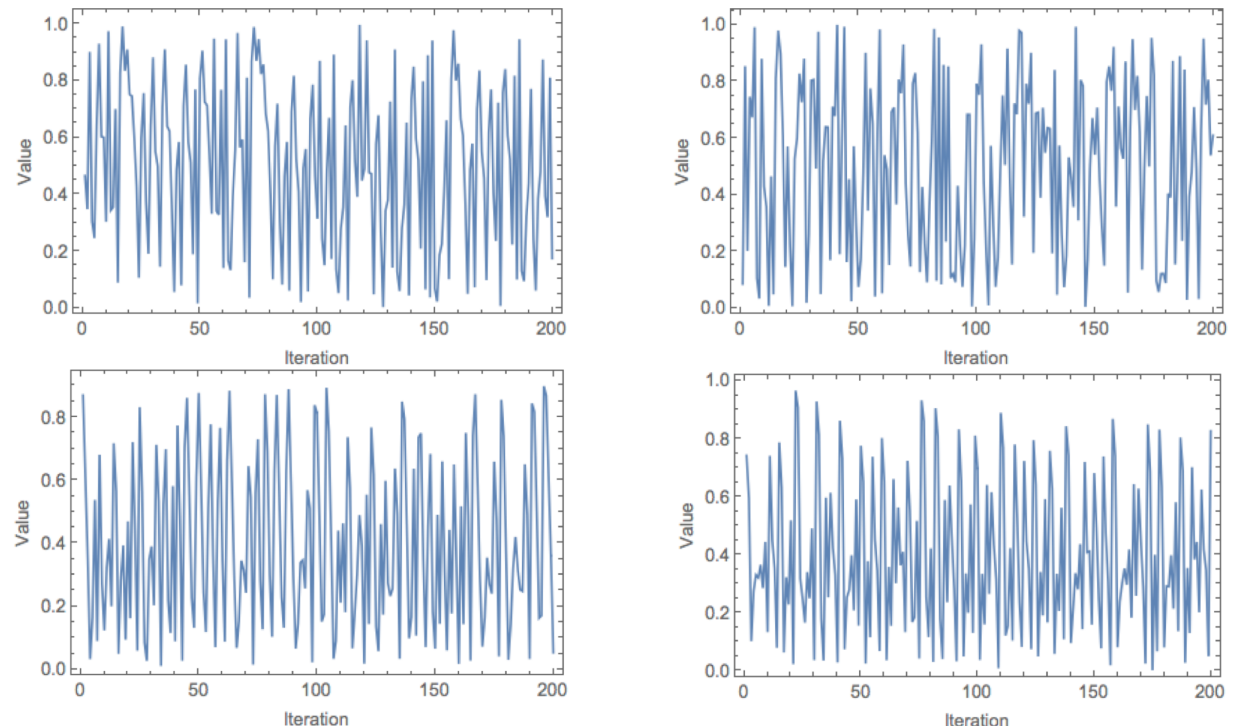

Fig. 5: Comparison of the dynamical sequencing of pseudo-random real numbers transferred into the range $<$ $0-1>$; upper left ? Arnold Cat map, upper right Dissipative standard map, bottom left Ikeda map, bottom right Lozi map. 

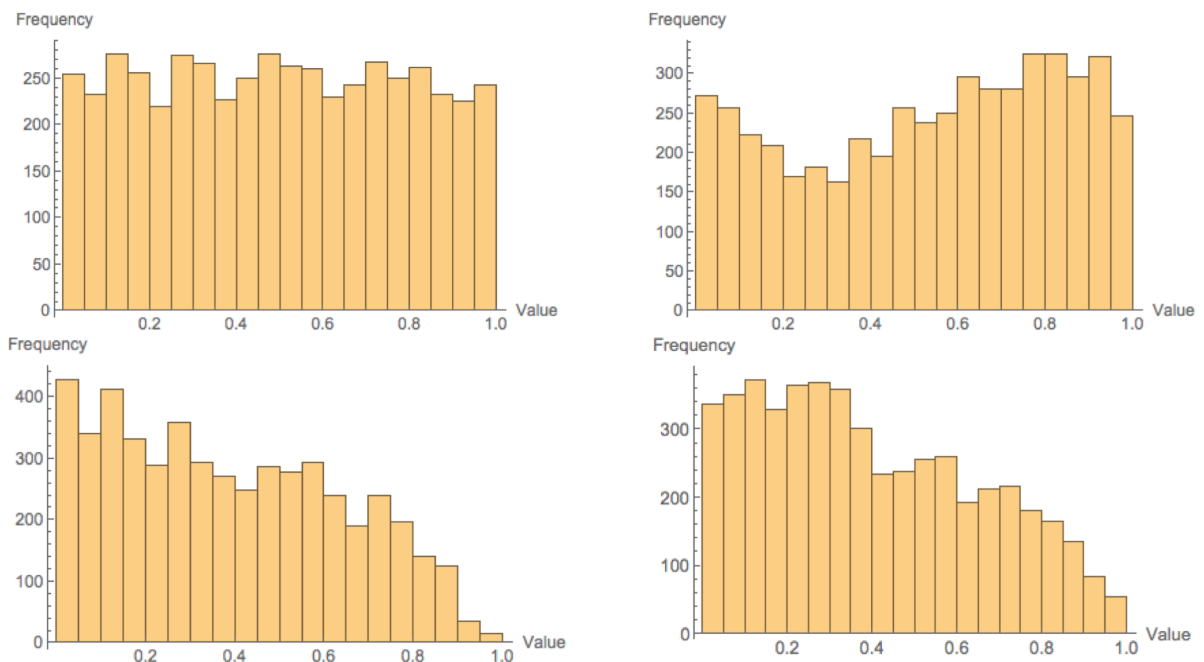

Fig. 6: Comparison of distributions of pseudo-random real numbers transferred into the range $<0-1>(5000$ samples); upper left - Arnold Cat map, upper right Dissipative standard map, bottom left Ikeda map, bottom right Lozi map.

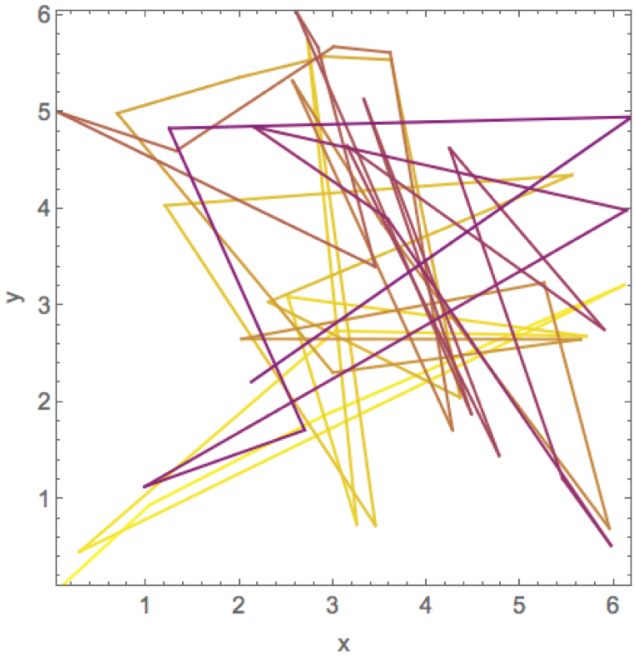

Fig. 7: Mapping of chaotic dynamics to the search space: Dissipative standard map - with default parameters $b=0.6$ and $k=8.8$. The coloring of path - from yellow to purple. A Small set of 50 iterations.

- Oscillators are giving more dynamical pseudo-random sequences with unique quasi-periodical sequencing in comparison with chaotic flows (See Fig. 1 and Fig. 2). Therefore chaotic oscillators are more suitable for CPRNG.

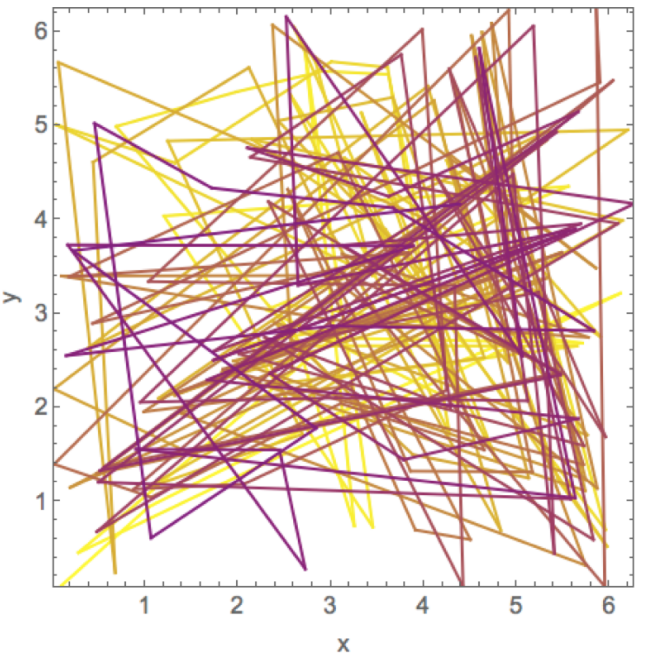

Fig. 8: Mapping of chaotic dynamics to the search space: Dissipative standard map - with default parameters $b=0.6$ and $k=8.8$. The coloring of path - from yellow to purple. A larger set of 200 iterations.

- Distributions of CPRNGs driven by timecontinuous systems are very similar.

- When comparing the time continuous systems and discrete maps - the first group is suitable only for direct CPRNG purposes 


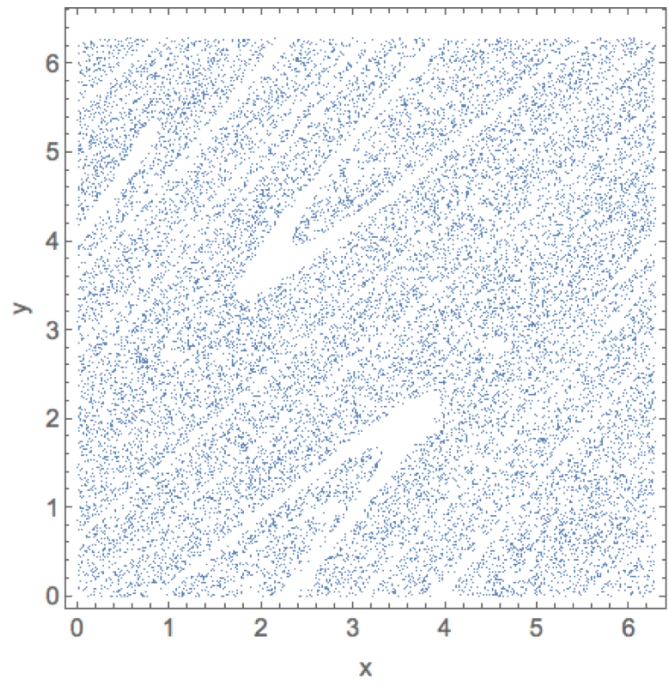

Fig. 9: Parametric plots of Dissipative standard map with changed parameters $b=0.9$ and $k=8.6$.

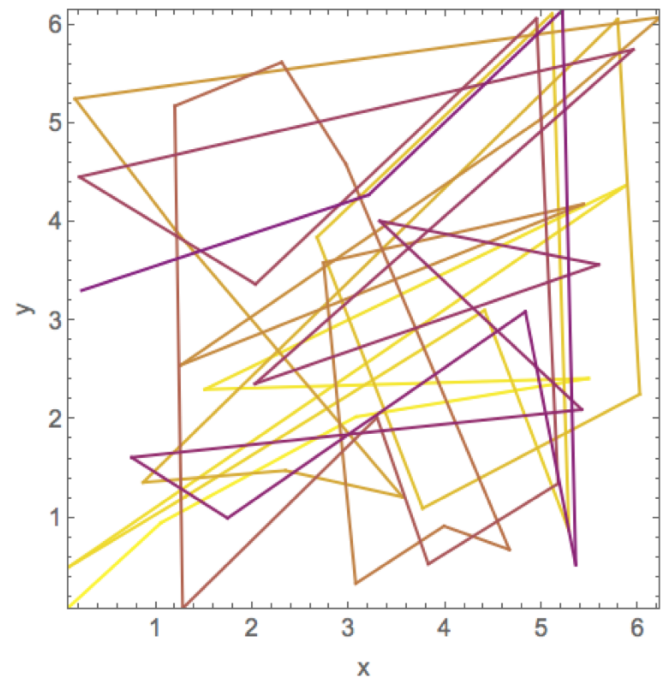

Fig. 10: Mapping of chaotic dynamics to the search space: Dissipative standard map - changed parameters $b=0.9$ and $k=8.6$. The coloring of path - from yellow to purple. A small set of 50 iterations.

(and preferably oscillators as stated in the previous point). Mapping of those systems to the optimization search space will lead only to covering a limited area, where the chaotic attractor is moving (See Fig. 1). Again, in the case of chaotic flows, these

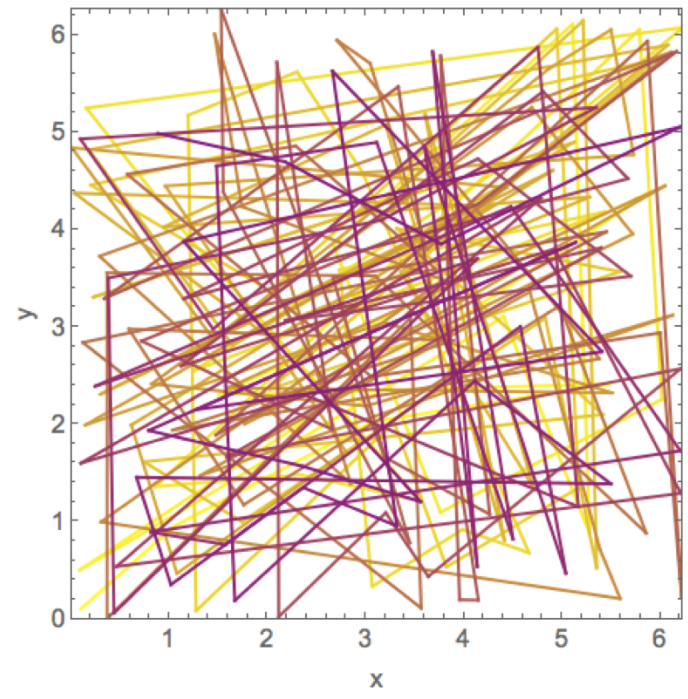

Fig. 11: Mapping of chaotic dynamics to the search space: Dissipative standard map - changed parameters $b=0.9$ and $k=8.6$. The coloring of path - from yellow to purple. A larger set of 200 iterations.

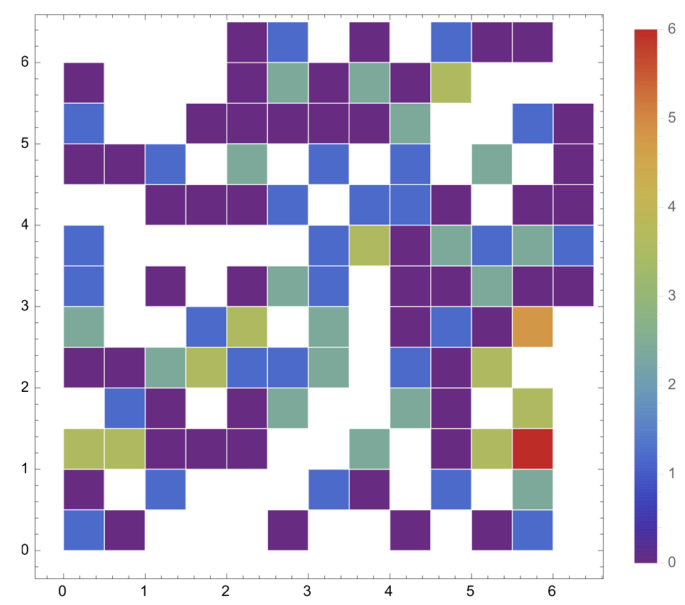

Fig. 12: Density histogram of mapping of chaotic dynamics to the search space; Dissipative standard map - with original parameters $b=0.6$ and $k=8.8$.

areas will be restricted only close to the cycles of the attractor. Oscillators are showing better coverage of the space. Contrary to chaotic flows and oscillators, the discrete maps show high potential for mapping 


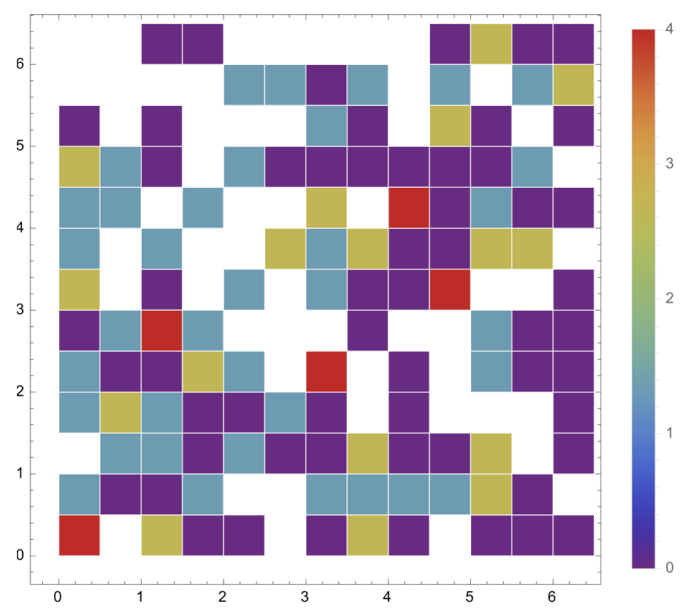

Fig. 13: Density histogram of mapping of chaotic dynamics to the search space; Dissipative standard map - with changed parameters $b=0.9$ and $k=8.6$.

Tab. 2: A brief summarization of experiment results.

\begin{tabular}{|c|c|c|c|}
\hline Usability & Flows & Oscillators & Maps \\
\hline CPRNG & N/A & YES & YES \\
\hline Mapping & NO & N/A & YES \\
\hline $\begin{array}{c}\text { Distribution change - } \\
\text { by parameters }\end{array}$ & NO & N/A & YES \\
\hline $\begin{array}{c}\text { Distribution change - } \\
\text { by sampling }\end{array}$ & NO & NO & - \\
\hline $\begin{array}{c}\text { Distribution change }- \\
\text { by different svstems }\end{array}$ & NO & NO & YES \\
\hline
\end{tabular}

or local search techniques. This ability is demonstrated in the previous section.

- Even though the coverage of search space is lower (Ikeda map) or very limited (Lozi map), these two chaotic maps can be combined within some hybrid multidimensional mapping.

- Furthermore, presented discrete chaotic maps generate different distributions of CPRNGs. Moreover, these systems have additional accessible parameters, which can by tuned and by changing of them, we can obtain even more CPRNG distribution classes.

\section{Conclusion}

The novelty of this research represents investigation on the utilization of the complex chaotic dynamics given by the several selected time-continuous chaotic systems, as the chaotic pseudo-random number generators and driving maps for the chaos based optimization (mapping of chaotic movement to the optimization/search space). This paper showed three groups of complex chaotic dynamics given by chaotic flows, oscillators and discrete chaotic maps.

Plans are including the testing of combination of the wider set of chaotic systems as well as the adaptive switching between systems, adaptive or chaos-like sequencing sampling rates and obtaining a large number of results to perform statistical tests.

\section{Acknowledgment}

This work was supported by Grant Agency of the Czech Republic - GACR P103/15/06700S, further by the Ministry of Education, Youth and Sports of the Czech Republic within the National Sustainability Programme project No. LO1303 (MSMT-7778/2014) and also by the European Regional Development Fund under the project CEBIA-Tech No. CZ.1.05/2.1.00/03.0089, and by Internal Grant Agency of Tomas Bata University under the project No. IGA/CebiaTech/2016/007.

\section{References}

[1] CELIKOVSKY, S. and I. ZELINKA. "Chaos Theory for Evolutionary Algorithms Researchers", in I. Zelinka, S. Celikovsky, H. Richter, G. Chen, Editors, Evolutionary Algorithms and Chaotic Systems, Springer, pp. 89-143, 2010.

[2] LEE, J. S. and K. S. CHANG. "Applications of chaos and fractals in process systems engineering", Journal of Process Control, vol. 6, no. 2?3, pp. 71-87, 1996. 
[3] WU J., J. LU and J. WANG. "Application of chaos and fractal models to water quality time series prediction", Environmental Modelling 65 Software, vol. 24, no. 5, pp. 632-636, 2009.

[4] LOZI, R. "Emergence of Randomness from Chaos", International Journal of Bifurcation and Chaos, vol. 22, no. 02, (2012).

[5] PERSOHN, K. J. and R. J. POVINELLI. "Analyzing logistic map pseudorandom number generators for periodicity induced by finite precision floating-point representation", Chaos, Solitons \& Fractals, vol. 45, no. 3, pp. 238-245, 2012.

[6] WANG, X.-Y. and W. X. QIN. "A new pseudo-random number generator based on CML and chaotic iteration", Nonlinear Dynamics, vol. 70, no. 2, pp. 1589-1592, 2012.

[7] YANG, L., X.-Y. WANG. "Design of Pseudo-random Bit Generator Based on Chaotic Maps", International Journal of Modern Physics B, vol. 26, no. 32, 2012.

[8] BUCOLO, M., R. CAPONETTO, L. FORTUNA, M. FRASCA, A. RIZZO. "Does chaos work better than noise?", Circuits and Systems Magazine, IEEE, vol. 2, no. 3, pp. 4-19, 2002.

[9] SENKERIK, R., M. PLUHACEK, A. VIKTORIN and Z. K. OPLATKOVA. "On The Simulation Of Complex Chaotic Dynamics For Chaos Based Optimization", in ECMS 2016 Proceedings, 2016, doi:10.7148/20160258 .

[10] AYDIN, I., M. KARAKOSE, E. AKIN. "Chaotic-based hybrid negative selection algorithm and its applications in fault and anomaly detection", Expert Systems with Applications, vol. 37, no. 7, pp. 5285-5294, 2010 .

[11] CAPONETTO, R., L. FORTUNA, S. FAZZINO, M. G. XIBILIA. "Chaotic sequences to improve the performance of evolutionary algorithms", IEEE Transactions on Evolutionary Computation, vol. 7, no. 3, pp. 289-304, 2003.
[12] ZHENYU, G, C. BO, Y. MIN, C. BINGGANG. "Self-Adaptive Chaos Differential Evolution" in L. Jiao, L. Wang, X.-b. Gao, J. Liu, F. Wu, Editors, Advances in Natural Computation, Springer, pp. 972-975, 2006.

[13] DAVENDRA, D., I. ZELINKA and R. SENKERIK. "Chaos driven evolutionary algorithms for the task of PID control", Computers 83 Mathematics with Applications, vol. 60, no. 4, pp. 1088-1104, 2010.

[14] SENKERIK, R., M. PLUHACEK, I. ZELINKA, Z. OPLATKOVA, R. VALA and R. JASEK. "Performance of Chaos Driven Differential Evolution on Shifted Benchmark Functions Set" in Proceedings of International Joint Conference SOCO?13-CISIS?13-ICEUTE?13, Springer International Publishing, pp. 41-50, 2014.

[15] LIANG, W., L. ZHANG, M. WANG. "The chaos differential evolution optimization algorithm and its application to support vector regression machine", Journal of Software, vol. 6, no. 7, pp. 1297-1304, 2011.

[16] COELHO, L. d. S., V. C. MARIANI. "A novel chaotic particle swarm optimization approach using Henon map and implicit filtering local search for economic load dispatch", Chaos, Solitons \&3 Fractals, vol. 39, no. 2, pp. 510-518, 2009.

[17] PLUHACEK, M., R. SENKERIK, D. DAVENDRA, Z. K. OPLATKOVA and I. ZELINKA. "On the behavior and performance of chaos driven PSO algorithm with inertia weight", Computers \& Mathematics with Applications, vol. 66, no. 2, pp. 122134, 2013.

[18] PLUHACEK, M., R. SENKERIK, I. ZELINKA. "Multiple Choice Strategy Based PSO Algorithm with Chaotic Decision Making - A Preliminary Study" in Proceedings of International Joint Conference SOCO'13-CISIS'13-ICEUTE13, Springer International Publishing, pp. 21-30, 2014. 
[19] BREST, J. "Self-adaptive control parameters? randomization frequency and propagations in differential evolution", Swarm and Evolutionary Computation, vol. 25, pp. 72-99, 2015.

[20] HAMAIZIA, T., R. LOZI. "Improving Chaotic Optimization Algorithm using a new global locally averaged strategy", Emergent Properties in Natural and Artificial Complex Systems, pp. 17-20, 2011.

[21] SPROTT, J. C. Chaos and Time-Series Analysis, Oxford University Press, 2003.

[22] BHARTI, L., M. YUASA. Energy Variability and Chaos in Ueda Oscillator. 2011. http://www.rist.kindai.ac.jp/no.23/yuasaEVCUO.pdf

[23] SENKERIK, R., M. PLUHACEK, D. DAVENDRA, I. ZELINKA, Z. K. OPLATKOVA. "Simulation Of TimeContinuous Chaotic UEDA Oscillator As The Generator Of Random Numbers For Heuristic", in ECMS 2015 Proceedings, 2015, doi:10.7148/2015-0543.

\section{About Authors}

Roman SENKERIK was born in the Czech Republic and went to the Tomas Bata University in Zlin, where he studied Technical Cybernetics and obtained his MSc degree in 2004, the Ph.D. degree in Technical Cybernetics in 2008 and Assoc. prof. in 2013 (Informatics). He is now an Assoc. prof. at the same university (research and courses in Evolutionary Computation, Applied Informatics, Cryptology, Artificial Intelligence, Mathematical Informatics).

Ivan ZELINKA was born in the Czech Republic and went to the Technical University of Brno, where he studied Technical Cybernetics and obtained his degree in 1995. He obtained the Ph.D. degree in Technical Cybernetics in 2001 at Tomas Bata University in Zlin. Now he is a full professor at the Technical University of Ostrava (research topics: Artificial Intelligence, Theory of Information, Computer security and viruses).

Michal PLUHACEK was born in the Czech Republic and went to the Faculty of Applied Informatics at Tomas Bata University in Zlin, where he studied Information Technologies and received his MSc degree in 2011 and the Ph.D. in 2016 with the dissertation topic: Modern method of development and modifications of evolutionary computational techniques. He now works as a researcher at the same university.

"This is an Open Access article distributed under the terms of the Creative Commons Attribution License, which permits unrestricted use, distribution, and reproduction in any medium, provided the original work is properly cited (CC BY 4.0)." 